\title{
The effect of meal size distribution and timing on interstitial glucose responses in healthy volunteers
}

\author{
C. Bellini, E. Dimidi, H. Chambers, M. Gibbs and S. Hampton \\ Faculty of Health and Medical Sciences, University of Surrey, Guildford, Surrey GU2 7XH, UK
}

There is a diurnal variation in postprandial glucose responses due to a progressive decrease in glucose intolerance and an increase insulin resistance through the day (Van Cauter et al. 1992; Service et al. 1983). Thus consuming a majority of food intake late in the day, compared to early in the day induces a less healthy metabolic response profile. In this study 24 hour glucose monitors have been utilised to assess glucose responses continually at one minute intervals to compare the complete 24 hour glycaemic profile when the same foods are consumed but in opposed temporal patterns. The aim is to assess the effects of meal size and time of day on glycaemic responses in healthy volunteers under real life conditions.

Seven healthy participants (3 M, $4 \mathrm{~F}$ ) mean age 21.9 years (1.46 SD) were recruited to take part in a randomised intervention crossover study. The study had of two meal sizes $(1200 \mathrm{kcals}, 400 \mathrm{kcals})$ and two distribution patterns. The lunch time meal was similar on both occasions (470 kcals). Leg 1 volunteers consumed a small breakfast meal (SBM) at 08:00 h followed by a set lunch (13:00 h) and a large evening meal (LEM) at 20:00 h: Leg 2 of the study the SBM and LEM were reversed. The calculated glycaemic index (GI) of the large meal was 76 and the small meal was 65 (Frost and Dornhurst 2000). Continuous glucose monitors (CGM) that monitor changes in interstitial glucose responses were worn for 5 days. Subjects underwent study legs on day 2 and 4 in a random order. Statistical analyses using repeated measures ANOVA (factors, time and treatment) was performed on individual glucose data and paired tests on total incremental area under curve (AUC) for glucose response over 24 hours (see histogram).

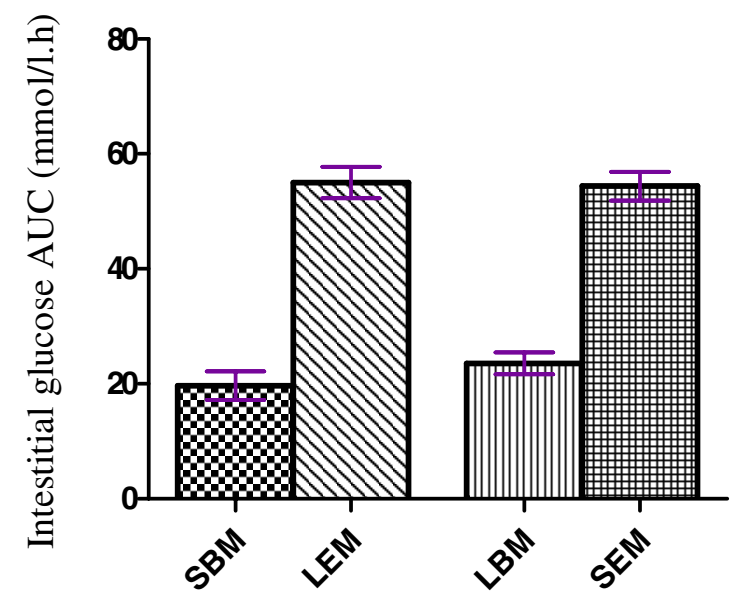

Meals

Statistical significances were found in postprandial glucose response between the same sized meals if consumed at $08: 00 \mathrm{~h}$ or $20: 00 \mathrm{~h}$ (Large meal $p=0.0002$, Small meal $p=0.0001)$. Two way RM ANOVA showed an effect of time $(p=0.0001)$ and meal distribution $(p=0012)$. There was no statistical significance in large or small distribution from morning to evening on 24 hour total AUC between the 2 legs (LEM 105 (SD) mmol/l.h, (LBM)106.6 (SD) mmol/l.h) [see histogram].

This study demonstrates that consuming meals with relatively high GI in the evening regardless of the calorific content results in significantly larger glycaemic responses than consuming the meal earlier in the day. Further investigation into meal distribution is required for dietary recommendations to be made particularly for those with impaired glucose responses.

1. Van Cauter E, Shapiro ET, Tillil H \& Polonsky KS (1992) The American Journal of Physiology 262(4): E467-E475.

2. Frost G \& Dornhorst A (2000) Diabetic Medicine: A Journal Of The British Diabetic Association 17(5): 336-345.

3. Service FJ, Hall LD, Westland RE, O'Brien PC, Go VL, Haymond MW \& Rizza RA (1983) Diabetologia 25(4): 316-321. 